\title{
Restricted autonomic flexibility in children with social phobia
}

\author{
Julian Schmitz, ${ }^{1}$ Martina Krämer, ${ }^{1}$ Brunna Tuschen-Caffier, ${ }^{1}$ \\ Nina Heinrichs, ${ }^{2}$ and Jens Blechert ${ }^{3}$ \\ ${ }^{1}$ Department of Clinical Psychology and Psychotherapy, University of Freiburg, Germany; ${ }^{2}$ Department of Clinical \\ Child and Adolescent Psychology and Psychotherapy, University of Bielefeld, Germany; ${ }^{3}$ Department of Psychology, \\ Stanford University, Stanford, California, USA
}

\begin{abstract}
Background: Psychophysiological hyperresponsiveness to social-evaluative stress plays a key role in current theories of social phobia (SP). Owing to the early onset of this disorder, the study of children with SP can help to improve etiological models. However, research to date has failed to clarify whether children with SP are physiologically hyperresponsive to stress or not. In addition, the existence of elevated pre-stressor baseline group differences complicates the interpretation of acute stress responses and therefore poses a challenge for this line of research. Drawing on current models of autonomic control to explain the relationship between baseline and stress responding in SP children and healthy controls (HC), we acquired a broad set of autonomic measures in our study. Method: To index baseline and stress reactivity and to assess autonomic flexibility, we assessed a comprehensive array of sympathetic and parasympathetic measures in SP children (aged 8 to 12 years; $n=30$ ) and healthy control children (HC, $n=26$ ), while exposing them to the Trier Social Stress Test for Children (TSST-C). Results: At baseline, the SP children showed higher levels of sympathetic (heart rate, electrodermal activity) and lower levels of parasympathetic (respiratory sinus arrhythmia, RSA) activation when compared to the HC children. During the TSST-C, the SP children showed similar HR responses but a limited RSA reactivity and a slower HR recovery relative to the HC children. Conclusion: Our study extends previous research by showing elevated baseline arousal and comparable stress responding in $\mathrm{SP}$ children relative to $\mathrm{HC}$ children. In addition, based on the autonomic flexibility model, we provide a potential explanation for the null findings of previous studies during stress. The pattern of elevated baseline heart rates and reduced RSA point to restricted autonomic flexibility in children with SP.

Keywords: Social phobia, children, autonomic flexibility, psychopathology.
\end{abstract}

\section{Introduction}

Social phobia (SP) in children is characterized by a persistent fear of humiliation and rejection by others, which leads to the avoidance of social situations (DSM-IV; APA, 1994). Prevalence rates of up to $7 \%$ place it among the most frequent mental disorders in children and adolescents, and the age of onset is as early as 8 years (Chavira, Stein, Bailey, \& Stein, 2004). SP causes significant impairment in social and academic functioning, and is a potential risk to normal development when it occurs at a young age (Beidel, Turner, \& Morris, 1999). The experience of psychophysiological arousal symptoms (e.g., heart racing, sweating) makes up a considerable proportion of the clinical manifestation and diagnostic criteria of SP (APA, 1994) and plays a key role in current theories on SP in adults (Clark \& Wells, 1995). Consequently, numerous studies have assessed psychophysiological responding to social stress in SP adult samples, yielding inconsistent results. While some studies found higher levels of heart rate (HR) before and during stress (Gerlach, Mourlane, \& Rist, 2004; Gerlach, Wilhelm, Gruber, \& Roth, 2001),

Conflict of interest statement: No conflicts declared. increased sympathetic and parasympathetic responding to social situations in female SPs or diagnostic subgroups (Grossman, Wilhelm, Kawachi, \& Sparrow, 2001; Hofmann, Newman, Ehlers, \& Roth, 1995), in other studies autonomic parameters did not differ between SPs and healthy controls (HC) (Edelmann \& Baker, 2002; Heiser, Turner, Beidel, \& Roberson-Nay, 2009). Thus, from these studies, to date it remains mostly unclear to what extent SP adults may differ from non-anxious individuals in their autonomic responses.

SP is known to have its onset during childhood (Wittchen, Stein, \& Kessler, 1999), which has motivated researchers to assess the role of psychophysiological responses in this age group. For example, in a study on a small sample of SP children, Beidel, Christ, and Long (1991) found increased subjective anxiety levels during a read-aloud task, but no HR difference in comparison to HCs. In addition to this study, there are only a few studies on samples with mixed anxiety disorders including SP or elevated levels of general anxiety which have assessed various parameters related to the autonomic nervous system (ANS) (Bakker, Tijssen, van der Meer, Koelman, \& Boer, 2009; Kagan, Reznick, \& Snidman, 1988; Monk et al., 2001; Yeragani, Rao, Pohl, Jampala, \& 
Balon, 2001). Monk et al. (2001) found an elevated baseline HR before and a blunted HR reactivity following a $\mathrm{CO}_{2}$ inhalation task in a large age-range sample of 9- to 18-year-old children and adolescents with mixed anxiety disorders including SP, compared to HCs. In addition, Yeragani et al. (2001) found higher levels of QT variability during rest in children with anxiety disorders including SPs relative to $\mathrm{HCs}$, which serves as an index for the activation of the sympathetic branch of the ANS.

These studies provide evidence that elevated levels of anxiety in childhood could be associated with autonomic dysregulation. However, the most consistent result appears to be a pattern of hyperarousal during baseline and an absence of differential reactivity to stress. In addition, the studies reviewed above suffer from several limitations. First, some studies used clinical samples that combined a variety of anxiety disorders, making specific conclusions about SP impossible. Second, the experimental tasks varied considerably and were partially not well validated. Third, many samples were characterized by a large age range, which is particularly troublesome due to findings of age-related changes in autonomic functioning (Wilhelm, Schneider, \& Friedman, 2006). Finally, most studies employed a limited array of psychophysiological measurements, capturing only some aspects of the multifaceted nature of autonomic functioning. To address these limitations, we acquired a comprehensive set of autonomic parameters during the Trier Social Stress Test for Children (TSST-C; Buske-Kirschbaum et al., 1997), a highly standardized and well-validated paradigm to elicit social-evaluative threat. Furthermore, we used a relatively homogeneous sample of children suffering from SP aged 8-12.

Most of the previous research has focused on cardiovascular responding to stress, implicitly equating elevated responding (e.g., heightened HR) to stress/ anxiety/psychopathology. However, this approach has variously been criticized as simplistic, and alternative psychophysiological concepts have been proposed. The concept of autonomic flexibility has been particularly useful to explain some of the contradictory findings in this field. According to this approach, a high $\mathrm{HR}$ as a response to a certain stressor would not necessarily be equated with stress/anxiety/psychopathology, but could indicate autonomic flexibility if the HR returns to baseline quickly afterwards (Beauchaine, 2001; Porges, 2007; Porges, Doussard-Roosevelt, Portales, \& Greenspan, 1996; Thayer \& Brosschot, 2005; Thayer \& Lane, 2000). Such swift HR adjustments are effectuated primarily by the parasympathetic nervus vagus, and respiratory sinus arrhythmia (RSA) is central to the autonomic flexibility concept as an indicator of vagal control.

Following this approach, a growing number of studies have indexed autonomic flexibility in children with various internalizing and externalizing disorders. Lower levels of tonic basal RSA and a corresponding blunted phasic RSA reactivity and recovery have been found to be an index for restricted vagal flexibility in children with various markers of psychopathology (El-Sheikh, Harger, \& Whitson, 2001; El-Sheikh \& Whitson, 2006; Greaves-Lord et al., 2010; Katz \& Gottman, 1997; Monk et al., 2001; Santucci et al., 2008). In SP children, the specific roles of RSA, RSA reactivity to social stress, and autonomic flexibility in general remain unclear, even though it is conceivable that lower basal parasympathetic activation may account not only for elevated tonic HR levels, which have been found in anxious children, but also for restricted HR responses to stressors (Kagan et al., 1988; Krämer et al., submitted; Monk et al., 2001).

Adopting the concept of autonomic flexibility, we aim to shed more light on tonic and phasic autonomic reactivity to social stress in SP and healthy children. We hypothesized that SP children would show restricted autonomic flexibility compared to HC children as represented by (1) lower RSA and a higher HR at baseline (Kagan et al., 1988; Monk et al., 2001), (2) reduced RSA and HR reactivity to the TSST-C (Greaves-Lord et al., 2010; Monk et al., 2001), and (3) slower HR and RSA recovery from the TSST (Santucci et al., 2008).

\section{Method \\ Participants}

Children aged 8-12 years were recruited from the community through an advertising campaign offering treatment and remuneration ( $€ 25$ - approx. $\$ 35$ - for parents and $€ 25$ in vouchers for children) for participation in a three-session study series. The study was approved by the ethics committee of the German Psychological Association. Complete data was available for 30 SP children (15 boys and 15 girls; $M$ [SD] age: 10.1 [1.24]) fulfilling a principal DSM-IV diagnosis of SP and 26 HC children (15 boys, 11 girls; $M$ [SD] age: 9.96 [1.14]) who did not meet diagnostic criteria for any lifetime Axis I disorder. SP children were excluded if SP was not the primary diagnosis or if they had a co-morbid DSM-IV diagnosis of severe depression, dysthymia or autism. Positive exclusion criteria for all children were medical conditions like asthma or use of medication that could alter physiological responses.

Following a telephone screening, children and parents gave informed consent for the diagnostic interview and were assessed by a PhD student and three advanced graduate clinical psychology students who had been specifically trained in the administration of the 'Kinder-DIPS' (Schneider, Unnewehr, \& Margraf, 2008). The Kinder-DIPS is a structured psychiatric interview that codes for mental disorders in children and adolescents according to criteria of both the International Classification of Mental and Behavioral Disorders (ICD10; WHO, 1992) and the DSM-IV (APA, 1994). All diagnostic sessions were videotaped and any uncertainties discussed and clarified with two experienced clinical psychologists. After the interview, children and 
their parents filled out several questionnaires on demographics and psychopathology and gave informed consent for the following experimental sessions. The following co-morbid conditions were found in the SP group: attention deficit hyperactivity disorder $(n=6)$, specific phobia $(n=4)$, oppositional-defiant disorder $(n=1)$, enuresis $(n=1)$, separation anxiety disorder $(n=1)$, and major depression (mild single episode; $n=1$ ).

\section{Procedure and experimental measures}

The current experiment was part of a larger two-center studies series on subjective, autonomic, endocrine, and behavioral characteristics of children with SP. The data presented below were obtained from only one of the study centers because certain cardiac and electrodermal parameters where only assessed at this site.

Following the diagnostic interviews, a laboratory session was scheduled. The 60-min laboratory session began between 3 and 4 pm for all children. Details on the procedure can be found in Krämer et al. (2011). In brief, after a baseline ( $5 \mathrm{~min}$ ), children were instructed about the upcoming TSST-C, consisting of preparation ( $5 \mathrm{~min}$ ) and two stress phases (speech, math, $5 \mathrm{~min}$ each, conducted in front of a two-person adult committee). In the speech task (termed TSST1), children were instructed to finish telling a story as excitingly as possible. Whenever children finished the story in less than 5 minutes, the observers asked them to continue ('There is time left, please continue telling the story!'). In the following mental arithmetic task (termed TSST2), children did a serial subtraction. The stressor was followed by three $10 \mathrm{~min}$ resting periods (labeled recovery 1-3) in which participating children watched pictures of landscapes on a computer screen for relaxation and answered several questionnaires on their perceived performance and cognitions during the tasks, but which were unrelated to autonomic parameters. During the lab session a total of seven subjective anxiety ratings $(0$ - no anxiety to 10 - extreme anxiety) were obtained.

\section{Psychometric measures}

The Social Anxiety Scale for Children - revised (SASC-R;(La Greca \& Stone, 1993). This instrument is designed to assess symptoms of social anxiety in children aged between 6 and 12 years. Factorial analyses of the German version of the SASC-R (Melfsen $\&$ Florin, 1997) revealed two factors: Fear of Negative Evaluation subscale (9 items) and Social Avoidance and Distress subscale (9 items). Total scores may range from 18 to 90. Like the original scale, the German version of the SASC-R shows satisfactory reliability $(r=.57-.81)$ and internal consistency $(\alpha=.63-.83)$. In our sample, FNE and SAD scale showed excellent internal consistency ( $\alpha=.87$ and $\alpha=.84$, respectively).

\section{Psychophysiological measures}

Electrodermal and cardiovascular channels were simultaneously recorded at $400 \mathrm{~Hz}$ using the VarioportII system (Becker Meditec, Karlsruhe, Germany), streamed to disk, and displayed on a PC monitor for online monitoring of data quality. Data inspection and manual artifact rejection were performed offline using ANSLAB (Wilhelm \& Peyk, 2005). The following psychophysiological indices were chosen to allow an assessment of both the sympathetical and parasympathetical branches of the ANS.

\section{Electrodermal measures}

Electrodermal activity was recorded from the middle phalanx of the index and middle finger of the left hand using 11-mm inner diameter $\mathrm{Ag} / \mathrm{AgCl}$ electrodes filled with isotonic electrode paste (TD-246, Med Associates, Inc., St. Albans, Vermont). Three parameters were calculated: skin conductance level, number of nonspecific skin conductance fluctuations (number of deflections from a zero-slope baseline exceeding .02 microsiemens), and amplitude of nonspecific skin conductance fluctuations. The electrodermal system is innervated primarily by the sympathetic branch of the ANS (Boucsein, 1992). Three SP children did not show any measurable electrodermal activity and were therefore excluded as non-responders (Lindstrom, 1996).

\section{Cardiovascular measures}

For the analyses the cardiac interbeat interval (IBI), calculated from ECG lead II as the interval in milliseconds between successive R-waves, was used. For presenting results IBI was converted to heart rate (HR; in beats per minute [bmp]) but all statistical analyses where based on IBI values (Quigley \& Berntson, 1996). Specific care was taken to exclude artifactual R-spikes in the ECG since these might bias estimates of HR variability parameters (Berntson, Quigley, Jang, \& Boysen, 1990).

Three measures were calculated beat-by-beat from the ECG and the finger pulse waveform measured by a plethysmographic sensor (Becker Meditec, Karlsruhe, Germany): (a) T-wave amplitude, calculated in reference to the isoelectric ECG baseline; (b) pulse wave transit time, as time between steepest upstroke and ECG $\mathrm{R}$-wave; and (c) pulse wave amplitude, as peak minus trough. All three measures primarily reflect cardiac sympathetic activity (Kline, Ginsburg, \& Johnston, 1998; Rau, 1991; Szabo, 1993).

Respiratory sinus arrhythmia (RSA in $\mathrm{ms}$ ) was calculated using complex demodulation (Hayano et al., 1994; Wilhelm, Grossman, \& Roth, 2005). In contrast to spectral analysis, complex demodulation provides timedependent changes in an instantaneous amplitude of the oscillatory component within a given frequency range. Thus, CDM is better suited to assess levels of RSA during acute mental or physiological stress, when the assumption of stationary for the spectral analysis is violated. For assessing the magnitude of RSA, we demodulated the amplitude of the IBI oscillation in a high frequency band (.15-.50 Hz), representing vagal control (Mezzacappa et al., 1997). Since RSA can be influenced by respiration (Grossman \& Taylor, 2007), respiration rate was measured using a respiration belt around the thorax of the participating children. Since the stressor involved speaking, valid data from the respiration channel was obtained only during the baseline period (Wilhelm, Handke, \& Roth, 2003). 


\section{Data reduction and statistical analyses}

Preprocessed psychophysiological measures were averaged in one-minute intervals. These intervals were then averaged within each of the seven experimental phases (Baseline, Preparation, TSST1, TSST2 - 5min each; Recovery 1-3 - 10 min each). Outliers ( $z$-values of $\geq \pm 3$ ) were excluded channel by channel. ${ }^{1}$ During statistical analysis two steps were taken to protect against spurious findings as a result of multiple tests: First, to obtain more reliable indices of sympathetic cardiovascular activity, the three cardiovascular measures pulse wave transit time, T-wave amplitude, and pulse wave amplitude were combined by $z$-transformation (within individuals) and averaging (between individuals) into a cardiovascular sympathetic index (CSI; cf. Blechert, Michael, Grossman, Lajtman, \& Wilhelm, 2007) (Cronbach's alpha of CSI $=.839$ ). The CSI was reversed because the three measures are inversely related to sympathetic activation. A similar approach was chosen for electrodermal activity: skin conductance level, number of nonspecific skin conductance fluctuations, and amplitude of nonspecific skin conductance fluctuations were combined to form an electrodermal sympathetic index (ESI, $\alpha=.941)$.

Second, a multivariate analysis of variance (MANOVA) including all five primary measures preceded univariate analyses for individual measures. This MANOVA (and follow-up univariate ANOVAs) included the factors Group (SP, HC) and Phase (baseline, preparation, TSST1, TSST2, recovery 1-3) to determine global group differences on the primary measures (Subjective anxiety, HR, ESI, CSI, and RSA). Global group effects were then decomposed by measure and by phase in univariate, repeated measures ANOVAs for the individual measures. To differentiate tonic state and phasic reactivity, baseline scores were separately submitted to between-group $t$-tests. Difference from baseline was then calculated for each of the TSST-C phases. Figure 1 shows an illustration of the analysis pattern.

To decompose the Phase factor, we distinguished between reactivity (baseline, preparation, TSST1) and recovery (TSST2, recovery 1-3) (cf. Kudielka et al., 2004) in separate Group $(\mathrm{SP}, \mathrm{HC}) \times$ Phase ANOVAs $^{2}$ (Greenhouse-Geisser adjustments). In general, Phase effects on physiological and subjective parameters were very strong, indicating robust reactivity to - and recovery from - the TSST-C. Thus, for the sake of brevity we will report only main effects or interactions of Group.

Cohen's $f$ is reported as effect-size measure for MANOVA and ANOVA results, Cohen's $d$ for $t$-tests. ${ }^{3}$ For a correlation analysis, which evaluated possible relations between baseline levels of sympathetic and parasympathetic activity with HR reactivity and recovery, two scores for HR reactivity (HR during TSST1 phase - HR

\footnotetext{
${ }^{1}$ Excluded outliers: RSA (1SP; $\left.1 \mathrm{HC}\right)$; CSI (4SP;1HC); ESI (4SP).

${ }^{2}$ In line with the results of previous research (Kudielka et al., 2004) we evaluated sex and age as covariates. Covariates had no substantial effects on the results and were omitted. Since baseline scores were correlated with group, they were not included as a covariate in the following repeated measures ANOVAs (Miller \& Chapman, 2001).

${ }^{3}$ Small effects $f>.10$; medium effects $f>.25$; large effects $\mathrm{f}>.40$ according to Cohen (1988).
}

Tonic and phasic analysis pattern

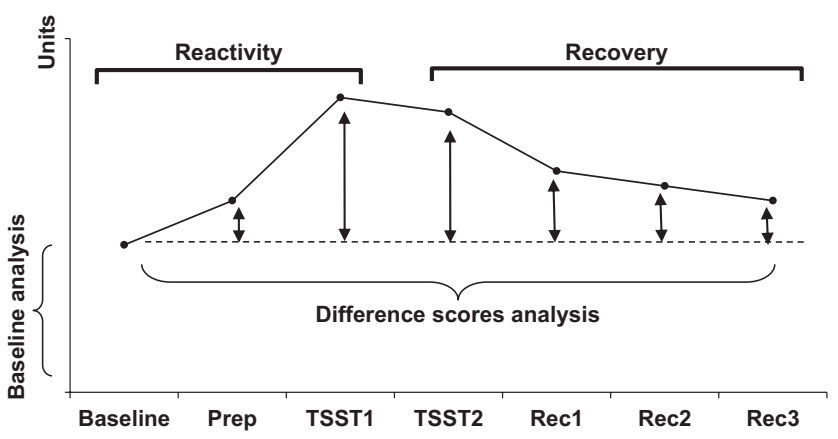

Figure 1 Tonic and Phasic Analysis Pattern

during baseline phase) and HR recovery (HR during recovery 3 phase - HR during TSST2 phase) were computed. The significance level for all analyses was set at $\alpha=.05$.

\section{Results \\ Participants' characteristics and psychometrics}

As shown in Table 1, groups where successfully matched on age, gender and education.

\section{General analysis of primary measures}

Figure 2 shows the baseline and difference scores for all primary measures. The MANOVA on the combined primary measures (anxiety, HR, RSA, CSI and ESI) revealed a significant Group $\times$ Phase interaction, $F(4,40)=7.22, p<.001, f=.81$, but no main effect of Group, $F(1,43)=2.67, p=.135, f=.23$.

\section{Subjective anxiety}

An independent sample $t$-test revealed higher anxiety scores during baseline in the SP group when compared to HC children, $t(54)=2.60, \quad p=.001$, $d=1.70$. The ANOVA for reactivity showed main effects of Group, $F(1,54)=6.48, p=.014, f=.34$, and a Group $\times$ Phase Interaction, $F(2,108)=4.21$, $p=.017, f=.27$. Inspections of the means in

Table 1 Participant characteristics

\begin{tabular}{|c|c|c|c|}
\hline & Social phobia & Healthy controls & \\
\hline & $M(S D)$ & $M(S D)$ & Statistic \\
\hline $\mathrm{n}$ in sample & 30 & 26 & \\
\hline Age (years) & $10.1(1.24)$ & $9.96(1.14)$ & $t(54)=0.43$ \\
\hline$\%$ Female & 50.0 & 42.3 & $\chi 2(1)=0.33$ \\
\hline $\begin{array}{l}\text { \% Primary } \\
\text { school }\end{array}$ & 50.0 & 34.6 & $\chi 2(1)=1.34$ \\
\hline SASC-R & $47.2(10.7)$ & $26.7(6.51)$ & $t(54)=8.43^{* * *}$ \\
\hline
\end{tabular}

Note: SASC-R = The Social Anxiety Scale for Children - revised. $M=$ mean; $S D=$ standard deviation.

${ }^{* *} p<.01 ; * * * p<.001$. 
(a)

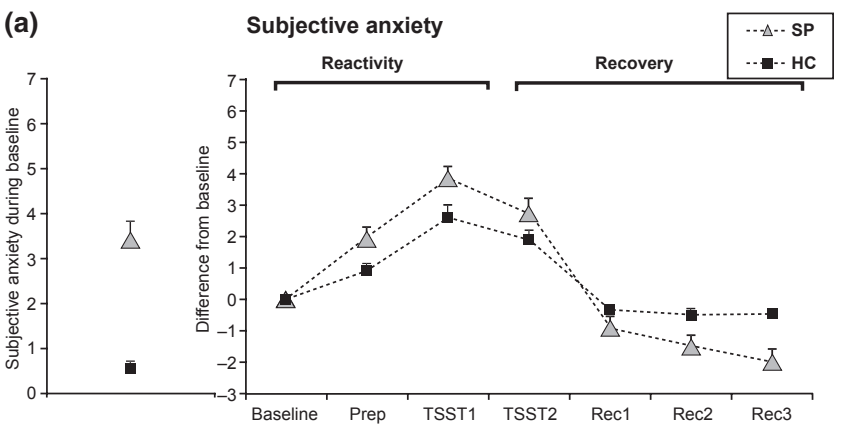

(b)

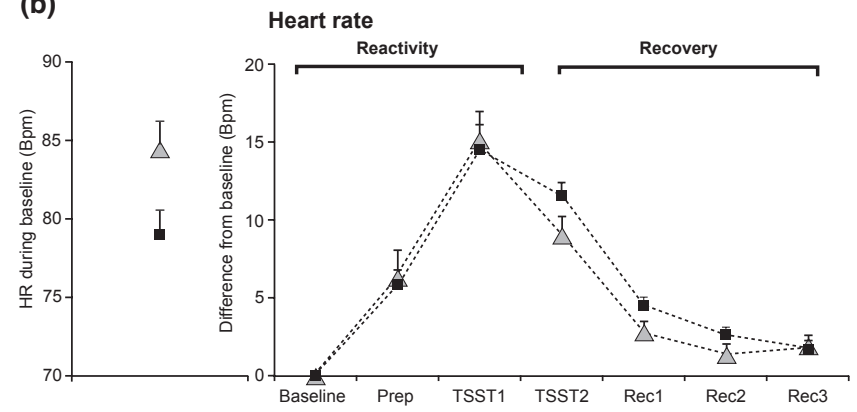

(c)

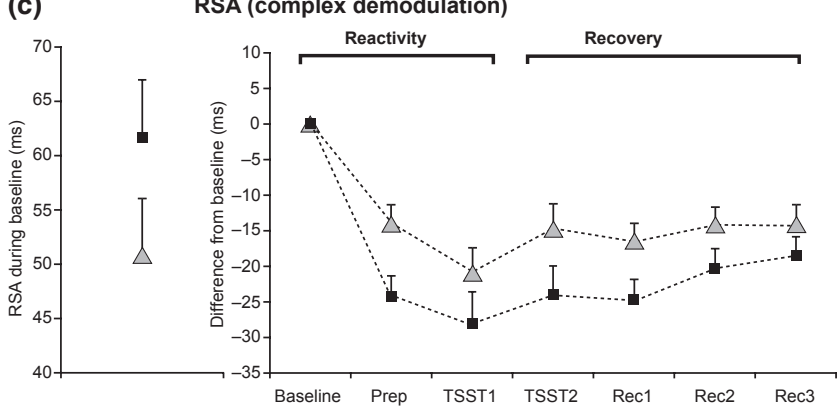

(d)

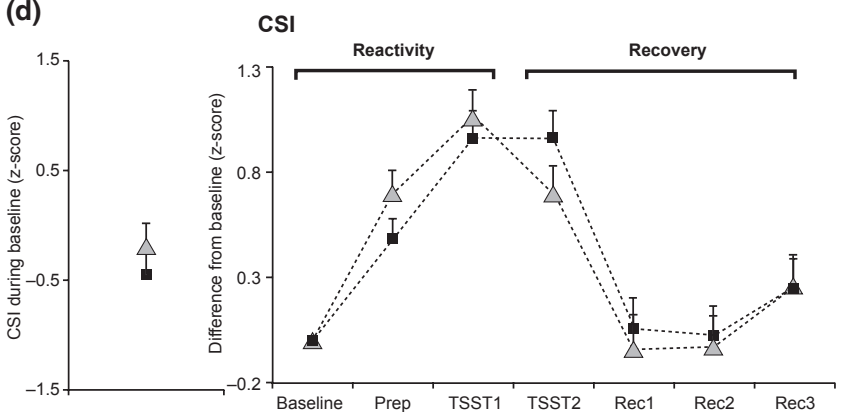

(e)

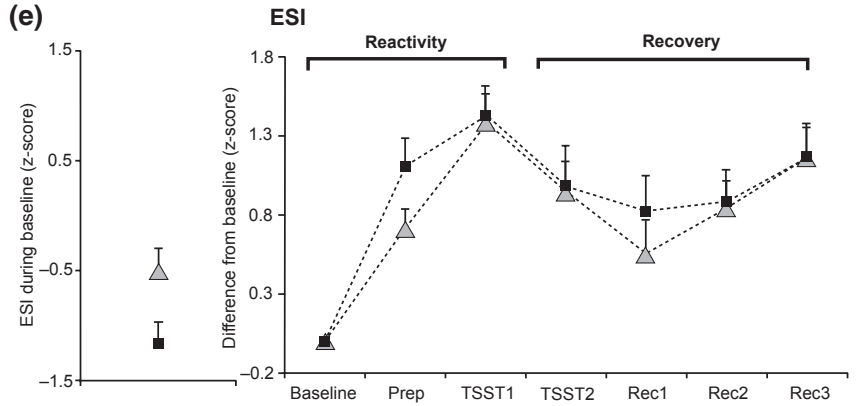

Figure 2 Baseline values, reactivity, and recovery on (a) subjective anxiety, (b) heart rate, (c) RSA, (d) CSI, and (e) ESI

Figure 1a suggests a stronger linear increase (Group $\times$ Phase linear trend $p=.021$, quadratic and higher trends, $p \mathrm{~s}>.221$ ) in subjective ratings in the SP group. The recovery ANOVA failed to show a main effect of Group, $F(1,54)=2.33, p=.132, f=.20$, but revealed a Group $\times$ Phase Interaction, $F(3,162)=$ 9.87, $p<.001, f=.36$. Inspections of the means in Figure 2A suggest a stronger decrease (linear trend, $p<.001$, quadratic and higher trends, $p \mathrm{~s}>$.099) in subjective anxiety ratings in the SP group.

\section{Heart rate}

The $t$-test for baseline HR differences revealed higher $\mathrm{HR}$ in the in the SP group when compared to HCs, $t(54)=2.06, p=.044, d=.55$. The reactivity ANOVA did not reveal Group main effects or interactions, Fs $<.40, p s>.527$. The recovery ANOVA yielded a significant effect for Group, $F(1,54)=4.24, p=.044, f=$ .24 , and its interaction with Phase, $F(3,162)=3.22$, $p=.024, f=.24$, which was mainly accounted for by a linear trend, $p=.016$, and a quadratic trend, $p=.003$. Inspection of the means in Figure 2B sug- gest a slower recovery of HR from the TSST-C in the SP group. ${ }^{4}$

\section{Cardiovascular and Electrodermal Sympathetic Index}

On the ESI scores, SP children showed an elevated baseline level when compared to $\mathrm{HCs}, t(50)=2.34$, $p=.024, d=.64$, but groups did not differ in their CSI scores, $t(49)=1.05, p=.298, d=.30$. The reactivity and recovery ANOVAs for the ESI or CSI scores did not reveal any significant effects involving Group, Fs $<2.45, p s>.124$, see Figures 2D and E.

\section{RSA}

SP children showed lower baseline RSA than HC children $t(54)=2.23, p=.030, d=.67$. The RSA reactivity ANOVA did not reveal a main effect of

\footnotetext{
${ }^{4}$ Figure 2 suggests that HR in both groups recovered from TSST1 to TSST2; however, both groups showed a robust reactivity from the short interval between both tasks to TSST2, thus we measured recovery starting with TSST2.
} 
Table 2 Correlation analysis

\begin{tabular}{|c|c|c|c|c|c|c|}
\hline & HR_base & HR_reac & HR_reco & RSA_base & CSI_base & ESI_base \\
\hline HR_base & 1 & & & & & \\
\hline HR_reac & $-.557^{* *}$ & 1 & & & & \\
\hline RSA_base & $-.643^{* *}$ & $.474^{* *}$ & $-.313^{*}$ & 1 & & \\
\hline CSI_base & $.279 *$ & .059 & -.023 & .106 & 1 & \\
\hline ESI_base & .037 & -.016 & .055 & -.062 & -.119 & 1 \\
\hline
\end{tabular}

Note . base $=$ baseline phase; reac $/$ reco $=$ reactivity $/$ recovery.

${ }^{*} p<.05 ;{ }^{* *} p<.01$.

Group, $F(1,52)=3.25, p=.077, f=.25$, but a Group $\times$ Phase interaction, $F(2,104)=3.68, \quad p=.028$, $f=.26$, accounted for mainly by a quadratic trend, $p<.001$, linear and higher trends, $p s>.145$. Inspections of the means in Figure $2 \mathrm{C}$ suggest a weaker RSA decrease in SP children. For recovery, there were no significant effects involving Group, Fs $<2.88$, ps $>.095 .^{5}$

\section{Correlation analysis}

In line with our assumption that the baseline autonomic state would determine HR stress responding and that HR stress responses would primarily be parasympathetically mediated, we calculated correlations in all participants between baseline, reactivity and recovery parameters (see Table 2). Across groups, higher baseline HR was associated with lower reactivity and faster recovery, demonstrating the baseline dependency of this measure. However, the specific nature of this baseline dependency is revealed by considering sympathetic and parasympathetic indices: lower levels of baseline RSA were associated with lower HR reactivity and slower HR recovery. ${ }^{6}$ Basal sympathetic activation was unrelated to reactivity or recovery, suggesting that phasic HR responses to the TSST were driven primarily by the parasympathetic withdrawal.

\section{Discussion}

The aim of our study was to investigate the role of autonomic functioning and its relation to autonomic flexibility during a social-evaluative stressor in SP children compared to a group of HC children. We hypothesized that SP children, relative to $\mathrm{HC}$ children, would show restricted autonomic flexibility as

\footnotetext{
${ }^{5}$ Even though baseline respiration rate did not significantly differ between groups, it was added to the analysis of baseline RSA as covariate since it has been shown that respiration rate itself influences RSA independent of vagal activity (Grossman $\&$ Taylor, 2007). However, the inclusion of this variable did not alter the results.

${ }^{6}$ RSA baseline levels were negatively related to HR baseline levels, which is consistent with the understanding that RSA indicates parasympathetic activity. Regarding the sympathetic ANS branch, higher CSI activity went along with higher HR baseline levels, as expected from its sympathetic origin.
}

represented by (1) lower baseline RSA and a higher baseline HR, (2) blunted RSA and HR reactivity to the TSST-C, and (3) slower HR and RSA recovery. In line with hypothesis 1 , the SP children showed lower RSA and higher HR levels during baseline. In addition, we found a stronger sympathetic activation in the SP children during baseline. Both elevated tonic HR levels and sympathetic activation have previously been reported by studies on children with mixed anxiety disorders or inhibited temperament (e.g., Bakker et al., 2009; Kagan et al., 1988; Monk et al., 2001; Yeragani et al., 2001). Regarding hypothesis 2, we found the expected blunted RSA reactivity to the TSST-C in the SP children but no group differences in HR reactivity, which confirms hypothesis 2 partially. Partially confirming hypothesis 3 , SP children were characterized by a slow HR recovery from the stressor.

How can we understand the underlying mechanisms of this pattern of autonomic responding? Two psychophysiological concepts may provide clarification. First, the autonomic flexibility approach emphasizes response flexibility: Rather than focusing on absolute HR, and equating high HR with pathology, proponents of this model consider HR increases as a healthy response to stress, if followed by swift returns to baseline. This response pattern, while energy efficient, facilitated fast adaptation to changing environments. Since such rapid changes in HR levels are effectuated by the parasympathetic $n$. vagus, its index RSA is equated with autonomic flexibility (Beauchaine, 2001; Porges, 2007; Porges et al., 1996; Thayer \& Brosschot, 2005; Thayer \& Lane, 2000). Second, since baseline group differences in the context of clinical anxiety are frequent phenomena, and measures such as the HR are strongly baseline-dependent, researchers need to give careful thought to physiological ceiling/floor effects before interpreting reactivity differences. Berntson and co-authors have revised the previously widely accepted law of initial values (Wilder, 1962) by postulating several autonomic modes (Berntson, Cacioppo, \& Quigley, 1991; Berntson, Cacioppo, Quigley, \& Fabro, 1994). Specifically, they assume that the sympathetic and parasympathetic branches can operate in coupled modes (e.g., reciprocal: activation of one branch causes deactivation of the other branch) or uncoupled modes (activation or deactivation of either branch is independent of the other 
branch). Most importantly, physiological ceiling or floor effects of an effector organ are dependent on the autonomic mode. In the present context, with SP children being characterized by elevated baseline HR levels, the assessment of both ANS branches can illuminate the underlying autonomic mode. Our results suggest that elevated baseline HR levels in SP arise from a reciprocal mode of decreased parasympathetic (lower RSA) and increased sympathetic activity (increased electrodermal activity and HR). This autonomic mode at baseline predicts restricted flexibility for further HR increases in the SP group. Consistent with this prediction, we found reduced RSA reactivity to the TSST and comparable HR increases despite subjective hyperreactivity in SP. This pattern is also evident when looking at correlations in the entire group: baseline HR and RSA levels were negatively related to TSST reactivity and recovery. Baseline sympathetic activity, by contrast, was unrelated to TSST reactivity and recovery.

In general, our study provides first evidence that SP in children may be associated with restricted autonomic flexibility in response to social-evaluative stress. That lower levels of basal RSA and a blunted RSA reactivity and recovery may indeed be maladaptive and negatively associated with measures of self-regulation in children, such as emotion regulation, has been shown repeatedly (e.g., Gentzler, Santucci, Kovacs, \& Fox, 2009; Santucci et al., 2008; Shibagaki \& Furuya, 1997; Suess, Porges, \& Plude, 1994). However, the question whether there might be a specific link between RSA reactivity in SP children and constructs of self-regulation needs further investigation. From a clinical point of view, tonically elevated arousal at the advent of a social performance situation might draw attention away from important situational cues, thereby impairing actual or perceived performance.

The following limitations may apply. Even though our results speak for restricted autonomic flexibility in SP children, further evidence is needed to show that our results hold for larger samples of SP children matched to HCs for other potential confounds such as IQ, reading, comprehension, and verbal fluency. From our data it remains unclear whether our findings are the cause or consequence of the disorder since our participants were already affected at the time of the study. Nevertheless, there are several findings which support the assumption that restricted autonomic flexibility may exist before the onset of psychopathology. First, higher basal HR levels, which might be due to lower tonic RSA levels, were found in preschool children with inhibited temperament and at risk of the development of SP (Kagan et al., 1988). In addition, a longitudinal study by Greaves-Lord et al. (2010) found that RSA reactivity in 10- to 12-year-old girls was predictive for increased levels of anxiety two years later. Second, both externalizing and internalizing problems in children have been found to be associated with basal levels of RSA and RSA reactivity (El-Sheikh et al., 2001; El-Sheikh \& Whitson, 2006; Katz \& Gottman, 1997). However, more longitudinal studies and prospective family designs, e.g., examining high risk samples of children from mothers with the diagnosis of SP, are needed to understand the exact role which autonomic flexibility plays in the development of psychopathology and its specificity for particular disorders (Beauchaine, 2001). In general our results emphasize the need for ambulatory assessment studies in order to evaluate whether the baseline differences we found in our sample were caused by anticipatory anxiety in the experimental situation. Indeed, some characteristics of anxiety disorders which have been replicated in the laboratory several times do not hold when investigated in an ambulatory context (e.g., Pfaltz, Michael, Grossman, Blechert, \& Wilhelm, 2009). Third, as shown by previous research, RSA levels may be affected by respiratory influences independent of changes in vagal control (Grossman \& Taylor, 2007). The involvement of speaking during our stressor made a concomitant assessment of respiratory parameters impossible. Consequently, it remains uncertain if the reduced RSA reactivity in SP reflects genuine group differences in parasympathetic activity or rather group differences in metabolic demands, tidal volume or respiratory rate (see Grossman \& Taylor, 2007 for a detailed discussion of non-parasympathetic influences on RSA).

\section{Conclusion}

In sum, our study provides first evidence that restricted autonomic flexibility may be characteristic of SP in children, which might partially explain the absence of group differences in reactivity or even reduced reactivity in SP. Regarding treatment implications our results could support cognitivebehavioral concepts for the treatment of childhood $\mathrm{SP}$, which have been found to be related to reductions in autonomic dysregulation in anxious adults (Friedman, 2007). More studies are needed to replicate our findings and to provide clarification regarding the question of the role that autonomic flexibility plays in the early development of the disorder, whether this pattern of autonomic response may be distinctive for SP and how it relates to other constructs of psychopathology, such as social competence or difficulties in emotion regulation.

\section{Acknowledgements}

This research was funded by the German Research Foundation (Grant TU 78/5-1; HE3342/4-2).

\section{Correspondence to}

Julian Schmitz, Department for Clinical Psychology and Psychotherapy, Engelbergerstrasse 41, 79106 Freiburg, Germany; Tel: +497612035485; Fax: +497612033022; Email: julian.schmitz@psychologie.uni-freiburg.de 


\section{Key points}

- Bodily symptoms are assumed to play a key role in the maintenance of SP.

- Restricted autonomic flexibility has been linked to psychopathology in children.

- Comprehensive assessment of psychophysiological measures in a homogeneous sample of SP children, exposed to a validated social stressor.

- SP children show restricted autonomic flexibility in terms of a higher tonic autonomic arousal during baseline and a blunted autonomic reactivity.

- SP children may show restricted autonomic flexibility which may be linked to other clinically relevant problems of self-regulation such as emotion regulation.

\section{References}

APA. (1994). Diagnostic and statistical manual of mental disorders (4th edn). Washington, DC: Author.

Bakker, M.J., Tijssen, M.A., van der Meer, J.N., Koelman, J.H., $\&$ Boer, F. (2009). Increased whole-body auditory startle reflex and autonomic reactivity in children with anxiety disorders. Journal of Psychiatry Neuroscience, 34, 314-322.

Beauchaine, T. (2001). Vagal tone, development, and Gray's motivational theory: Toward an integrated model of autonomic nervous system functioning in psychopathology. Development and Psychopathology, 13, 183-214.

Beidel, D.C., Christ, M.G., \& Long, P.J. (1991). Somatic complaints in anxious children. Journal of Abnormal Child Psychology, 19, 659-670.

Beidel, D.C., Turner, S.M., \& Morris, T.L. (1999). Psychopathology of childhood social phobia. Journal of the American Academy of Child and Adolescent Psychiatry, 38, 643-650.

Berntson, G.G., Cacioppo, J.T., \& Quigley, K.S. (1991). Autonomic determinism: The modes of autonomic control, the doctrine of autonomic space, and the laws of autonomic constraint. Psychological Review, 98, 459-487.

Berntson, G.G., Cacioppo, J.T., Quigley, K.S., \& Fabro, V.T (1994). Autonomic space and psychophysiological response. Psychophysiology, 31, 44-61.

Berntson, G.G., Quigley, K.S., Jang, J.F., \& Boysen, S.T. (1990). An approach to artifact identification: Application to heart period data. Psychophysiology, 27, 586-598.

Blechert, J., Michael, T., Grossman, P., Lajtman, M., \& Wilhelm, F.H. (2007). Autonomic and respiratory characteristics of posttraumatic stress disorder and panic disorder. Psychosomatic Medicine, 69, 935-943.

Boucsein, W. (Ed.). (1992). Electrodermal activity. New York: Plenum.

Buske-Kirschbaum, A., Jobst, S., Wustmans, A., Kirschbaum, C., Rauh, W., \& Hellhammer, D. (1997). Attenuated free cortisol response to psychosocial stress in children with atopic dermatitis. Psychosomatic Medicine, 59, 419-426.

Chavira, D.A., Stein, M.B., Bailey, K., \& Stein, M.T. (2004). Child anxiety in primary care: Prevalent but untreated. Depression and Anxiety, 20, 155-164.

Clark, D.M., \& Wells, A. (Eds.). (1995). A cognitive model of social phobia. New York: Guilford Press.

Cohen, J. (1988). Statistical power analysis for the behavioral sciences (2nd edn). Hillsdale, NJ: Erlbaum.

Edelmann, R.J., \& Baker, S.R. (2002). Self-reported and actual physiological responses in social phobia. British Journal of Clinical Psychology, 41, 1-14.

El-Sheikh, M., Harger, J., \& Whitson, S.M. (2001). Exposure to interparental conflict and children's adjustment and physical health: The moderating role of vagal tone. Child Development, 72, 1617-1636.

El-Sheikh, M., \& Whitson, S.A. (2006). Longitudinal relations between marital conflict and child adjustment: Vagal regulation as a protective factor. Journal of Family Psychology, 20, 30-39.
Friedman, B.H. (2007). An autonomic flexibility-neurovisceral integration model of anxiety and cardiac vagal tone. Biological Psychology, 74, 185-199.

Gentzler, A.L., Santucci, A.K., Kovacs, M., \& Fox, N.A. (2009). Respiratory sinus arrhythmia reactivity predicts emotion regulation and depressive symptoms in at-risk and control children. Biological Psychology, 82, 156-163.

Gerlach, A.L., Mourlane, D., \& Rist, F. (2004). Public and private heart rate feedback in social phobia: A manipulation of anxiety visibility. Cognitive Behaviour Therapy, 33, 36-45.

Gerlach, A.L., Wilhelm, F.H., Gruber, K., \& Roth, W.T. (2001). Blushing and physiological arousability in social phobia. Journal of Abnormal Psychology, 110, 247-258.

Greaves-Lord, K., Tulen, J., Dietrich, A., Sondeijker, F., van Roon, A., Oldehinkel, A., et al. (2010). Reduced autonomic flexibility as a predictor for future anxiety in girls from the general population: The TRAILS study. Psychiatry Research, 179, 187-193.

Grossman, P., \& Taylor, E.W. (2007). Toward understanding respiratory sinus arrhythmia: Relations to cardiac vagal tone, evolution and biobehavioral functions. Biological Psychology, 74, 263-285.

Grossman, P., Wilhelm, F.H., Kawachi, I., \& Sparrow, D. (2001). Gender differences in psychophysiological responses to speech stress among older social phobics: Congruence and incongruence between self-evaluative and cardiovascular reactions. Psychosomatic Medicine, 63, 765-777.

Hayano, J., Mukai, S., Sakakibara, M., Okada, A., Takata, K., \& Fujinami, T. (1994). Effects of respiratory interval on vagal modulation of heart rate. American Journal of Physiol, 267(1 Pt 2), H33-40.

Heiser, N.A., Turner, S.M., Beidel, D.C., \& Roberson-Nay, R. (2009). Differentiating social phobia from shyness. Journal of Anxiety Disorders, 23, 469-476.

Hofmann, S.G., Newman, M., Ehlers, A., \& Roth, W.T. (1995). Psychophysiological differences between subgroups of social phobia. Journal of Abnormal Psychology, 104, 224-231.

Kagan, J., Reznick, J.S., \& Snidman, N. (1988). Biological bases of childhood shyness. Science, 240, 167-171.

Katz, L.F., \& Gottman, J.M. (1997). Buffering children from marital conflict and dissolution. Journal of Clinical Child Psychology, 26, 157-171.

Kline, K.P., Ginsburg, G.P., \& Johnston, J.R. (1998). T-wave amplitude: Relationships to phasic RSA and heart period changes. International Journal of Psychophysiology, 29, 291-301.

Krämer, M., Seefeld., W., Heinrichs, N., Tuschen-Caffier, B., Schmitz, J., Wolf, O., \& Blechert, J. (2011). Subjective, autonomic, and endocrine reactivity during social stress in children with social phobia. Manuscript in preparation.

Kudielka, B.M., Buske-Kirschbaum, A., Hellhammer, D.H., \& Kirschbaum, C. (2004). Differential heart rate reactivity and recovery after psychosocial stress (TSST) in healthy children, younger adults, and elderly adults: The impact of age and gender. International Journal of Behavioral Medicine, 11, $116-121$. 
La Greca, A.M., \& Stone, W.L. (1993). Social Anxiety Scale for Children Revised: Factor structure and concurrent validity. Journal of Clinical Child Psychology, 22, 17-27.

Lindstrom, L.H. (1996). Clinical and biological markers for outcome in schizophrenia: A review of a longitudinal followup study in Uppsala schizophrenia research project. Neuropsychopharmacology, 14(3 Suppl.), 23S-26S.

Melfsen, S., \& Florin, I. (1997). Ein Fragebogen zur Erfassung sozialer Angst bei Kindern (SASC-R-D). Kindheit und Entwicklung, 6, 224-229.

Mezzacappa, E., Tremblay, R.E., Kindlon, D., Saul, J.P., Arseneault, L., Seguin, J., et al. (1997). Anxiety, antisocial behavior, and heart rate regulation in adolescent males. Journal of Child Psychology and Psychiatry, 38, 457-469.

Miller, G.A., \& Chapman, J.P. (2001). Misunderstanding analysis of covariance. Journal of Abnormal Psychology, $110,40-48$

Monk, C., Kovelenko, P., Ellman, L.M., Sloan, R.P., Bagiella, E., Gorman, J.M., et al. (2001). Enhanced stress reactivity in paediatric anxiety disorders: Implications for future cardiovascular health. International Journal of Neuropsychopharmacology, 4, 199-206.

Pfaltz, M.C., Michael, T., Grossman, P., Blechert, J., \& Wilhelm, F.H. (2009). Respiratory pathophysiology of panic disorder: An ambulatory monitoring study. Psychosomatic Medicine, 71, 869-876.

Porges, S.W. (2007). The polyvagal perspective. Biological Psychology, 74, 116-143.

Porges, S.W., Doussard-Roosevelt, J.A., Portales, A.L., \& Greenspan, S.I. (1996). Infant regulation of the vagal 'brake' predicts child behavior problems: A psychobiological model of social behavior. Developmental Psychobiology, 29, 697-712.

Quigley, K.S., \& Berntson, G.G. (1996). Autonomic interactions and chronotropic control of the heart: Heart period versus heart rate. Psychophysiology, 33, 605-611.

Rau, H. (1991). Responses of the T-wave amplitude as a function of active and passive tasks and beta-adrenergic blockade. Psychophysiology, 28, 231-239.

Santucci, A.K., Silk, J.S., Shaw, D.S., Gentzler, A., Fox, N.A., \& Kovacs, M. (2008). Vagal tone and temperament as predictors of emotion regulation strategies in young children. Developmental Psychobiology, 50, 205-216.

Schneider, S., Unnewehr, S., \& Margraf, J. (2008). KinderDIPS: Diagnostisches Interview bei psychischen Störungen im Kindes- und Jugendalter (2nd edn). Göttingen: Hogrefe.

Shibagaki, M., \& Furuya, T. (1997). Baseline respiratory sinus arrhythmia and heart-rate responses during audi- tory stimulation of children with attention-deficit hyperactivity disorder. Perceptual and Motor Skills, 84(3 Pt 1), 967-975.

Suess, P.E., Porges, S.W., \& Plude, D.J. (1994). Cardiac vagal tone and sustained attention in school-age children. Psychophysiology, 31, 17-22.

Szabo, A. (1993). The combined effects of orthostatic and mental stress on heart rate, T-wave amplitude, and pulse transit time. European Journal of Applied Physiology and Occupational Physiology, 67, 540-544.

Thayer, J.F., \& Brosschot, J.F. (2005). Psychosomatics and psychopathology: Looking up and down from the brain. Psychoneuroendocrinology, 30, 1050-1058.

Thayer, J.F., \& Lane, R.D. (2000). A model of neurovisceral integration in emotion regulation and dysregulation. Journal of Affective Disorders, 61, 201-216.

WHO. (1992). The international classification of mental and behavioural disorders: Clinical descriptions and diagnostic guidelines. Geneva: Author.

Wilder, J. (1962). Basimetric approach (law of initial values) to biological rhythms. Annals of the New York Academy of Siences, 98, 1211-1228.

Wilhelm, F.H., Grossman, P., \& Roth, W.T. (2005). Assessment of heart rate variability during alterations in stress: Complex demodulation vs. spectral analysis. Biomedical Sciences Instrumentation, 41, 346-351.

Wilhelm, F.H., Handke, E.M., \& Roth, W.T. (2003). Detection of speaking with a new respiratory inductive plethysmography system. Biomedical Sciences Instrumentation, 39, 136141.

Wilhelm, F.H., \& Peyk, P. (2005). ANSLAB 4.0: Autonomic nervous system laboratory [software]. Available from http:// www.sprweb.org/repository/index.cfm.

Wilhelm, F.H., Schneider, S., \& Friedman, B.H. (2006). Psychophysiological assessment. In M. Hersen (Ed.), Clinican's handbook of child behavioral assessment. Amsterdam: Elsevier.

Wittchen, H.U., Stein, M.B., \& Kessler, R.C. (1999). Social fears and social phobia in a community sample of adolescents and young adults: Prevalence, risk factors and co-morbidity. Psychological Medicine, 29, 309-323.

Yeragani, V.K., Rao, K.A., Pohl, R., Jampala, V.C., \& Balon, R. (2001). Heart rate and QT variability in children with anxiety disorders: A preliminary report. Depression and Anxiety, 13, $72-77$.

Manuscript accepted 18 March 2011 